\title{
Clinical drug tests adapted for speed
}

\section{Flexible approach allows cancer researchers to change course mid-trial according to patient response.}

When cancer researchers gathered in Washington DC for a major conference last week, much of the buzz was not about the results of clinical trials, but about a new way of doing them. Seeking to avoid slow clinical trials and their frequently disappointing results, pharmaceutical companies are increasingly turning to 'adaptive' trials. These are revamped midstream, on the basis of early data, to focus on the patients for whom the drug shows hints of promise.

The approach has been controversial, but is catching on with both researchers and regulators as companies struggle to combat the nearly $50 \%$ failure rate of drugs in large, expensive late-stage trials. "At this rate, drug development is just not sustainable," says Michael Krams, head of the neurology franchise at Johnson \& Johnson in Collegeville, Pennsylvania.

Cancer-genome sequencing has revealed tumours to be wildly diverse, with even those of the same cancer type harbouring a wide range of mutations. This means that some therapies work in only a subset of patients. Too often, averaging the effects of a drug across all patients with a given cancer drowns out the small signal of a subpopulation for which there is a clinical benefit.

Two trials described at last week's annual meeting of the American Association for Cancer Research - a breast-cancer study called I-SPY 2 and a lung-cancer trial code-named BATTLE - address these issues by allowing researchers to analyse data in the middle of a trial. From this, they can determine which patients are responding to a given drug, and whether biomarkers such as specific mutations are linked to that response. The trial is then restructured, midcourse, to treat patients with the drug that best suits their specific biomarkers. I-SPY 2 is also designed for testing to stop if it becomes clear that the drug is not helping, a general ethical benefit to the approach.

In addition to being faster, and therefore cheaper, than traditional approaches, the strategy should make trials more sensitive to small

\section{"We are really hoping} to drive down the cost of clinical trials 50 -fold."

subpopulations of drug-responsive patients, says Laura Esserman, a surgeon at the University of California, San Francisco, and a lead investigator of I-SPY 2. "We are really hoping to drive down the cost of clinical trials 50-fold," she says. "Otherwise I don't think drug companies are going to be interested in taking the risk of developing a drug for these small numbers of patients."

The concept of adaptive clinical trials dates back to the 1970s, although it is a route that few investigators have followed given the traditional emphasis on adhering to proven trial designs. This may be changing: a survey of 16 pharmaceutical companies and statistical consultants involved in trial designs identified only three or fewer adaptive trials that began each year between 2003 and 2006, but in 2007, there were 13 (J. Quinlan et al. Clin. Trials 7, 167-173; 2010).

One reason for the shift is increasing guidance from regulators about what adaptive trial designs they deem acceptable. In 2007, the European Medicines Agency detailed its stance on the trials, and this February, the US
Food and Drug Administration $\overrightarrow{5}$ (FDA) released draft guidance for industry.

Improvements in statistical techniques have eased the way for adaptive trials, which, according to regulators, should involve advanced statistical modelling. Such experiments can also require as much as six months more pre-trial planning than standard clinical trials, says Krams. And access to statisticians with expertise in adaptive trials has been a limiting factor, says Lesley Seymour of the clinical trials group of the National Cancer Institute of Canada in Ontario.

Nevertheless, enthusiasm about adaptive trials has been building. It may even be outstripping reality, cautions Stuart Pocock, a medical statistician at the London School of Hygiene \& Tropical Medicine. "They have been hyped by some people, but we're still trying to determine what potential these trials have," he says.

Krams also notes that some investigators use the term 'adaptive' as an excuse to get away with doing shorter trials in fewer patients. "Unfortunately there are cowboys out there who abuse the term 'adaptive design' and use it as a tool to cut corners," he says.

Some regulators - including the FDA have also expressed concerns that even legitimate adaptive trials can compromise an experiment's integrity because of the requirement that data be unblinded and analysed midtrial. Changing the course of a trial midstream could allow investigators and participants to infer how the therapy is performing, possibly colouring their perception of the drug's efficacy and introducing bias. But Seymour says that there are ways to construct a trial to minimize this risk, and an independent statistician can be included on data-monitoring boards to prevent undue influence on data analysis by trial sponsors.

Ultimately, Pocock says, some of these details will have to be worked out as researchers gain more experience with adaptive trials. "We're all on a learning curve together," he says.

Heidi Ledford

See Editorial, page 1245. 